ARTICLE

\title{
Phase separation on cell surface facilitates bFGF signal transduction with heparan sulphate
}

Song Xue (1) 1,2,4, Fan Zhou ${ }^{1,4}$, Tian Zhao ${ }^{1,4}$, Huimin Zhao ${ }^{1}$, Xuewei Wang ${ }^{1}$, Long Chen (1) 1, Jin-ping Li ${ }^{2,3} \&$ Shi-Zhong Luo (i) ${ }^{1 凶}$

Liquid-liquid phase separation (LLPS) plays important roles in various cellular processes, facilitating membrane-less organelles construction, chromatin condensation, signal transduction on inner membrane and many other processes. Current perception is that LLPS relies on weak multivalent interactions and crowded environments intracellularly. In this study, we demonstrate that heparan sulfate can serve as a platform to induce the phase separation of basic fibroblast growth factor on cell surface. The phase separation model provides an alternative mechanism how bFGF is enriched to its receptors, therefore triggering the signaling transduction. The research provides insights on the mechanism how growth factors can be recruited to cell surface by heparan sulfate and execute their functions, extending people's view on phase separation from intracellular to extracellular proteins at cellular level.

\footnotetext{
${ }^{1}$ Beijing Key Laboratory of Bioprocess, College of Life Science and Technology, Beijing University of Chemical Technology, 100029 Beijing, China. ${ }^{2}$ Beijing Advanced Innovation Centre for Soft Matter Science and Engineering, Beijing University of Chemical Technology, 100029 Beijing, China. ${ }^{3}$ Department of Medical Biochemistry and Microbiology, Uppsala University, 75123 Uppsala, Sweden. ${ }^{4}$ These authors contributed equally: Song Xue, Fan Zhou, Tian Zhao.

凶email: luosz@mail.buct.edu.cn
} 
LPS plays critical roles in cellular processes, including the formation of membrane-less organelles, construction of stress granules, regulation of genome organisation and control of synaptic signalling ${ }^{1-3}$. LLPS driven by weak multivalent interactions promotes formation of distinct functional condensates inside cells ${ }^{2,4}$. Those condensates can be assembled in the cytoplasm and nucleoplasm, such as processing bodies involved in RNA turnover, stress granules reacting against harmful conditions, and chromatin organisation ${ }^{1,5}$. LLPS can also occur on plasma membrane or endoplasmic reticulum, which provides platforms for condensates formation, facilitating cell signalling ${ }^{6-8}$, tight junction formation and synaptic transmission $^{9-13}$. Most of current reported LLPS in vivo happens in the intracellular environment, which might be due to the high protein density in cells and other biomolecules are amenable to form multivalent weak interactions that drive phase separation ${ }^{1,4}$. As the mechanisms that promote and regulate LLPS in vitro and inside cells are getting elucidated ${ }^{14,15}$, it remains an open question whether LLPS can happen in extracellular environments. Here, we demonstrate that basic fibroblast growth factor (bFGF) undergoes LLPS, which is critical for its signalling activity.

The fibroblast growth factors (FGFs) are a family of proteins with key roles in variety of processes, such as embryonic development, tissue regeneration and wound healing ${ }^{16,17}$. The member of FGF2, also named basic FGF (bFGF) from its rich basic residuals, is an important regulator of cell growth and differentiation under physiological and pathological conditions ${ }^{16,17}$. In extracellular matrix (ECM), bFGF bounds with heparan sulphate proteoglycans (HSPGs) for storage and is released to cell surface by matrix degradation, serving as a mechanism in response to injury or tissue reorganisation ${ }^{18}$. bFGF can also be secreted by adipocytes, and then diffuses to nearby cells. Secreted and released bFGF diffuses to nearby cells and is sequestered by HSPG that are tethered to the cell surface. On responsive cells, bFGF forms a ternary complex by binding to HSPG and fibroblast growth factor receptor (FGFR), and triggers downstream response $^{18}$. It also has been proposed that HSPG can associate with bFGF with a low affinity in a dynamic manner to assist receptor binding in close proximity ${ }^{19}$.

Here, we show that the weak binding promotes bFGF to form a condensate with HSPG via phase separation, and this condensate can further incorporate FGFR to form the active receptor complex for signal transduction.

\section{Results and discussion}

Heparin promotes bFGF to undergo liquid-liquid phase separation. Proteins capable of phase separation usually contain intrinsically disordered regions or tandem repeats. bFGF contains disordered $\mathrm{N}$ - and C-terminal sequences ${ }^{20}$ and can assemble into oligomers $^{21}$, which may provide sufficient multivalent weak interactions for LLPS. To explore the possibility of phase separation, enhanced green fluorescent protein fused bFGF (eGFP-bFGF) was expressed from E. coli and purified (Supplementary Fig. 1a). This eGFP fused bFGF has similar activity to stimulate cell proliferation as the wild-type protein (Supplementary Fig. 1b). The status of purified protein at various concentrations of PEG-8000 was examined under confocal microscope. Protein droplets formed and enlarged as the concentration of PEG increase to $10 \%$, indicating LLPS. Further increase of PEG concentration led to a decrease in both the size and quantity of the droplets and an increase in irregular aggregation (Fig. 1a). Though the eGFP tag provides great convenience for observing the LLPS of bFGF, there may be some concerns about its effects on phase separation, due to its negatively charged properties and dimerisation tendency ${ }^{22,23}$. Negative controls of
eGFP alone were mixed with 10\% PEG and no phase separation was observed. The solution turbidity also peaked at $10 \%$ PEG concentration and dropped at lower or higher PEG concentration, which is consistent with the image-based analysis (Fig. 1b). The droplets exhibited typical liquid properties. In fluorescence recovery after photobleaching (FRAP), fluorescence of the droplets quickly recovered, suggesting that their contents have high fluidity (Fig. 1c). A subset of droplets also merged during observation, consistent with their liquid-like nature. In contrast, eGFP-bFGF formed solid aggregation in 20\% PEG, distinguished by the irregular morphology and irreversible FRAP results (Fig. 1d). The results demonstrated that bFGF tends to phase separate in a mild crowded environment but form aggregates if the environment is overcrowded. As the extracellular environment is generally less crowded than the cytosol ${ }^{24}$ and bFGF is present at a low concentration, the finding suggests that bFGF can phase separate under physiological conditions.

Though PEG and other crowding agents are commonly used to mimic crowded intracellular environments ${ }^{25}$, bFGF is extracellular and the environment is distinct. HSPGs are abundant in the ECM and cell surface and are known to interact with bFGF ${ }^{26}$. As the negatively charged liner polysaccharide can provide multiple bFGF binding sites and result in more multivalent interactions, we hypothesise that interaction between bFGF and HSPG may promote LLPS. To test this hypothesis, we mixed heparin, a commonly used HS mimetic, with $5 \mu \mathrm{M}$ eGFP-bFGF at different ratios and examined the behaviour of the mixture. As the molar ratio of heparin to eGFP-bFGF increased to 1:10, more and lager droplets were formed, indicating stronger phase separation (Fig. 1e), while eGFP alone remained homogeneous with heparin. The results suggested that heparin promotes the phase separation of bFGF. Higher concentrations of heparin prevented droplets formation, demonstrating a "reentrant" behaviour, similar to the effects of RNA in the phase separation of many RNA binding proteins 27,28 . Indeed, heparin or HS share similar structural properties with RNA, such as rich negative charges and linear repetitive structure, and may promote LLPS following a similar mechanism as RNA. The change in turbidity is also consistent with the observations under microscopy (Fig. 1f). These observations suggest that heparin promotes bFGF phase separation. It has been reported that binding to heparin can increase the stability of bFGF by protecting it from proteolytic degradation ${ }^{29,30}$. We further tested whether heparin-induced phase separation can increase bFGF thermostability, as bFGF tends to unfold quickly at body temperature. eGFP-bFGF was incubated under different temperatures with or without heparin. With increased temperature, bFGF alone formed aggregates, while the samples with heparin remained in the liquid phase at $42{ }^{\circ} \mathrm{C}$ (Supplementary Fig. 2). The results suggest that heparininduced phase separation increased thermostability of bFGF.

Considering the highly charged properties of bFGF and heparin, it is natural to assume that the LLPS was driven by electrostatic interactions. We monitored the heparin-induced phase separation of eGFP-bFGF under different salt concentrations (Supplementary Fig. 3) and found that the LLPS was inhibited at high salt concentrations. Additionally, another highly negatively charged compound, poly uracil (polyU) was examined for phase separation induction of bFGF. The polyU showed similar dose-dependent effects as heparin. Those results confirmed that electrostatic interactions are the driven force of the heparin-induced LLPS of bFGF.

Despite the charges, we would like to dig deeper on the structure basis of heparin-bFGF interaction for LLPS. Natural heparin is highly sulphated and the charged sulpho group is likely critical for the interaction with bFGF. It has been reported that 6O-sulfate groups is critical for HS to promote the activity of bFGF $^{31}$. To assess the importance of sulfation in heparin, we used 
a
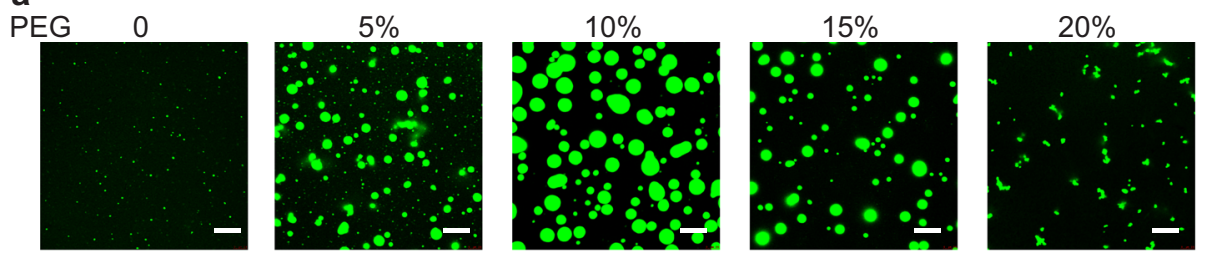

eGFP control

$10 \%$ PEG
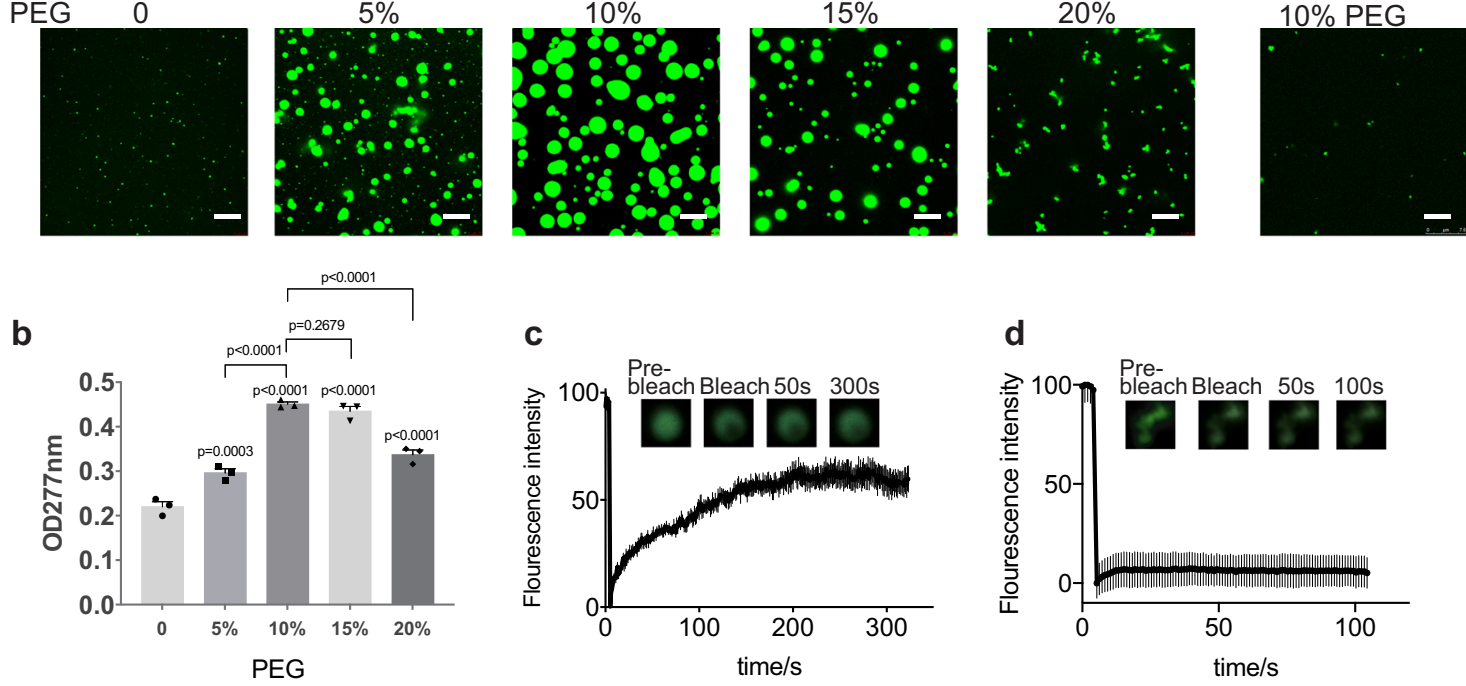

e
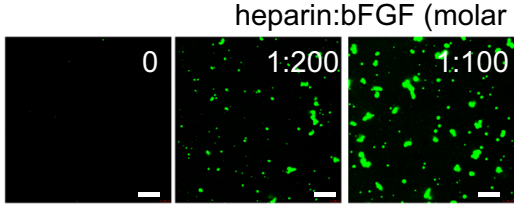

ratio)

heparin:eGFP=1:20
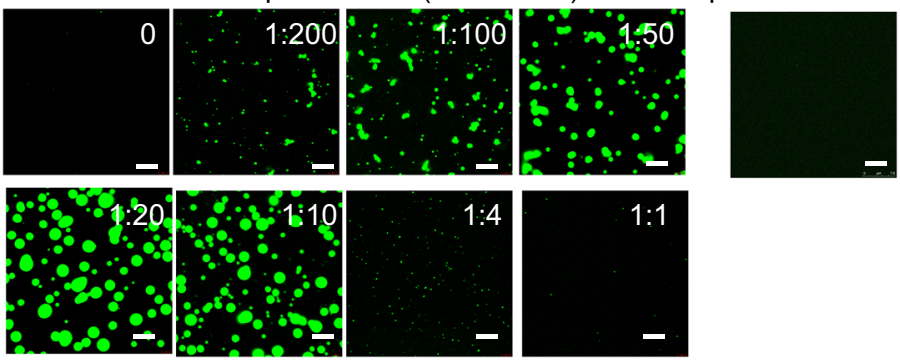

de-O-sulfated heparin:bFGF (molar ratio)

h

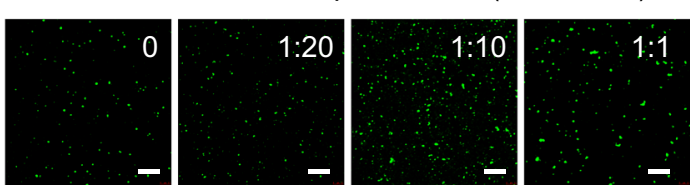

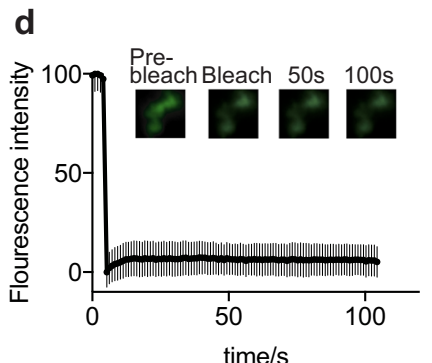

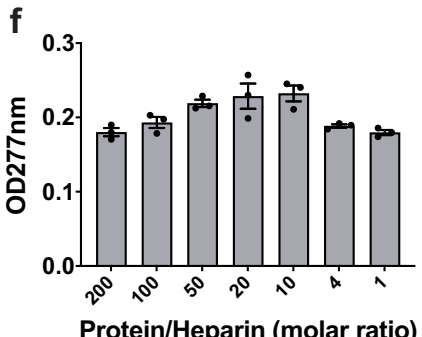

g

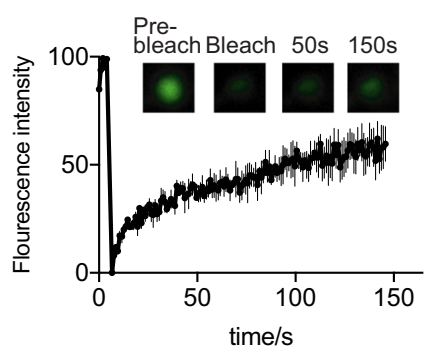

Fig. 1 bFGF undergoes phase separation in the presence of PEG or heparin. a Confocal microscopy images of assembling status of $5 \mu \mathrm{M}$ eGFP-bFGF with $0-20 \%$ PEG, with eGFP alone in 10\% PEG as control. Scale bar $=5 \mu \mathrm{m}$. b Turbidity measurement of eGFP-bFGF with $0-20 \%$ PEG. $n=3$ biologically independent samples, data are presented as mean values \pm SEM. Comparisons among groups were performed using ordinary one-way ANOVA test. c, FRAP of the droplets formed by eGFP-bFGF with 10\% PEG. $n=5$ biologically independent samples, data are presented as mean values \pm SEM. $\mathbf{d}$ FRAP of the aggregates formed by eGFP-bFGF with 20\% PEG. $n=3$ biologically independent samples, data are presented as mean values \pm SEM. e Confocal microscopy images of assembling status of $5 \mu \mathrm{M}$ eGFP-bFGF mixed with heparin at different ratios, with heparin: eGFP $=1: 20$ as control. Scale bar $=5 \mu$ m. f Turbidity measurement of eGFP-bFGF with heparin. $n=3$ biologically independent samples, data are presented as mean values \pm SEM. $\mathbf{g}$ FRAP results of the droplets formed by heparin:eGFP-bFGF $=1: 20 . n=3$ biologically independent samples, data are presented as mean values \pm SEM. $\mathbf{h}$ Confocal microscopy images of assembling status of $5 \mathrm{uM}$ eGFP-bFGF mixed with de-O-sulphated heparin at different ratios. Scale bar $=5 \mu \mathrm{m}$.

a de-O-sulphated heparin (OD-heparin) ${ }^{32}$. Addition of the ODheparin failed to induce LLPS of bFGF (Fig. 1h). This indicates that the phase separation of bFGF is mainly driven by the multivalent interactions between the protein and highly negatively charged sulpho group on heparin.

Meanwhile, we repeated the key experiments of LLPS with tagfree bFGF (Supplementary Fig. 4a, b). The results were consistent with the eGFP- version, confirming that bFGF can naturally undergo LLPS.

Oxidation status of bFGF affects its LLPS. Before exploring the behaviour of bFGF at the cellular level, we investigated the structural mechanisms of bFGF-heparin interaction. bFGF has two exposed cysteines and can exist as dimers and oligomers. The two exposed thiol groups form disulphide bonds under oxidising conditions and become unstable with aging of the protein ${ }^{33}$. Dimerisation is important to bFGF activity, which further induces FGFR dimerisation and activation ${ }^{21}$. Chemically conjugated multivalent bFGF was also reported to be more potent to stimulate cell proliferation since it is believed to bring more receptors in close proximity, assisting the dimerisation of FGFR for signalling transduction ${ }^{34}$. To examine whether reduction and oxidation of the cysteine residues in bFGF affect its phase separation, we added $1 \mathrm{mM}$ hydrogen peroxide or $5 \mathrm{mM} 1,4-$ Dithiothreitol (DTT) to the eGFP-bFGF solutions with various concentrations of heparin (Fig. 2a). Notably, phase separation of bFGF was essentially inhibited by DTT regardless with or without heparin. With hydrogen peroxide added, the bFGF alone formed 
a
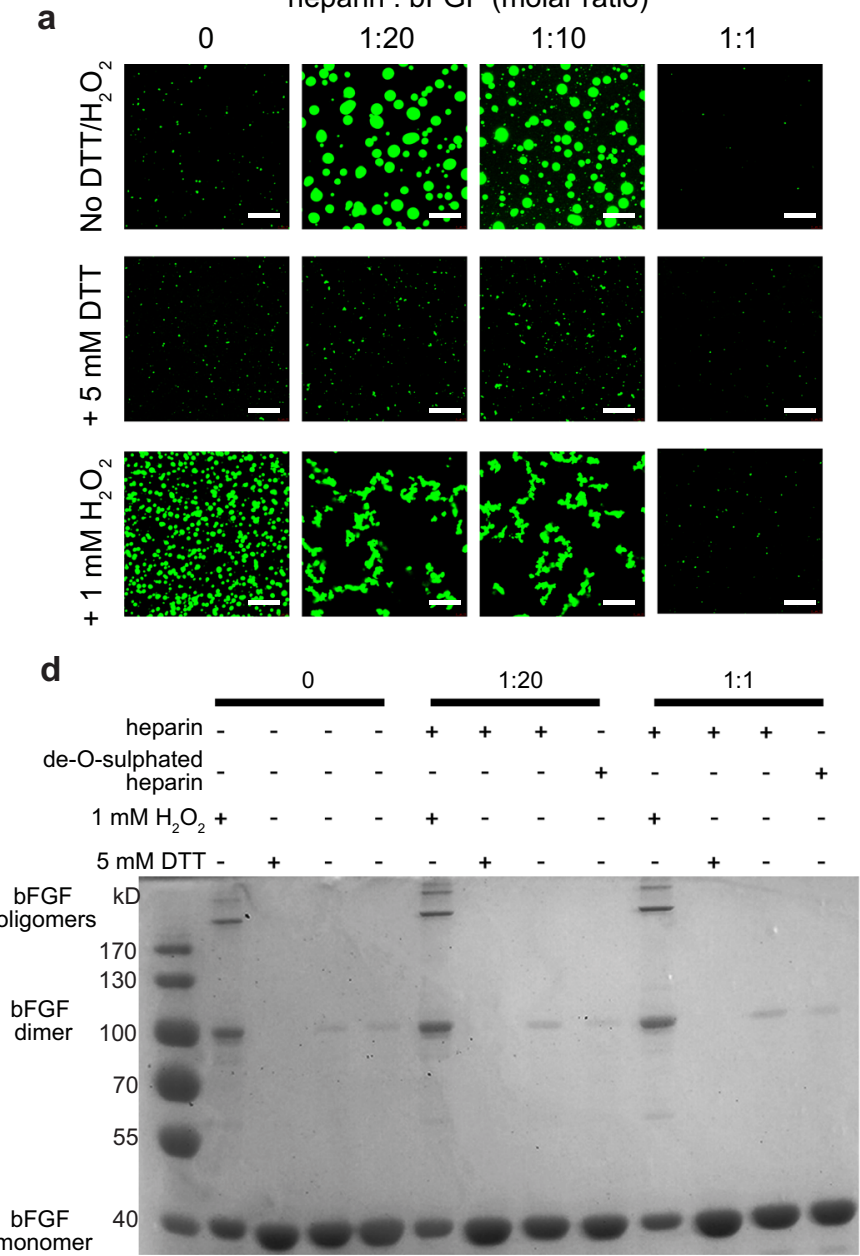
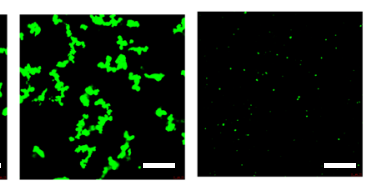

$1: 1$
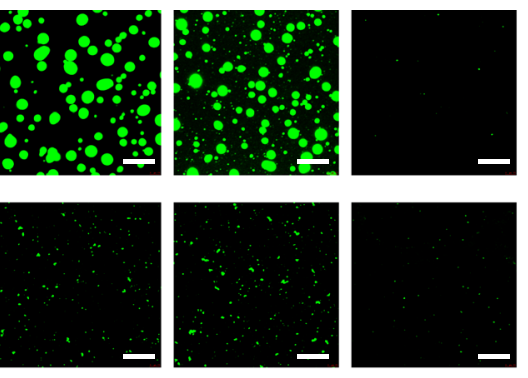

b

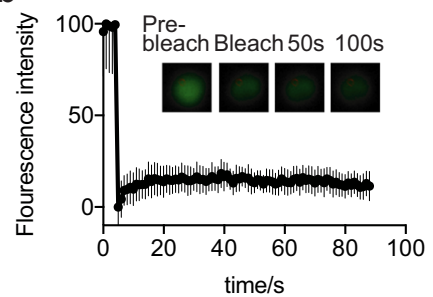

C
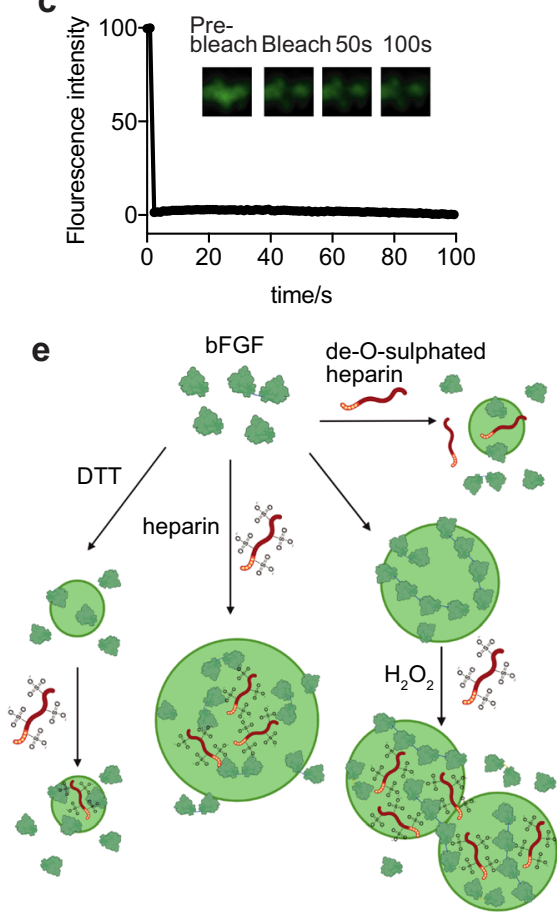

Fig. 2 Interaction of bFGF and heparin. a Confocal microscopy images of bFGF condensates with various ratios of heparin with $5 \mathrm{mM}$ DTT or $1 \mathrm{mM} \mathrm{H}_{2} \mathrm{O}_{2}$. Scale bar $=5 \mu \mathrm{m}$. b FRAP of the condensates formed by eGFP-bFGF oxidised in the presence of $1 \mathrm{mM}$ peroxide. $n=3$ biologically independent samples, data are presented as mean values \pm SEM. c FRAP of the condensates formed by oxidised eGFP-bFGF: heparin $=1: 20$. $n=4$ biologically independent samples, data are presented as mean values \pm SEM. d Non-reduced SDS-PAGE of the eGFP-bFGF with different oxidative conditions and heparin concentrations, showing its assembling status. e Schematic illustration of the proposed mechanism of bFGF assembling under different oxidative status with heparin/ de-O-sulphated heparin.

round condensates (Fig. 2a). However, FRAP assay showed no mobility of those condensates, indicating that they were gel-like entities crosslinked by disulphide bonds (Fig. 2b). The round shapes of the condensates implied that phase separation may occur in early stage and the droplets solidified quickly. Addition of heparin promoted the formation of clusters of condensates having no mobility either (Fig. 2c). This indicates that the oxidative condition resulted in larger population of dimers or even oligomers as confirmed by SDS-PAGE (Fig. 2d), which possesses more multivalent interactions of bFGF and heparin, favourable for aggregates formation. Endogenous bFGF exists mainly in monomers, with a small portion of dimers and multimers. We have reasons to believe that the population of different assembling status of bFGF in physiological conditions can provides sufficient multivalency for phase separation but not for aggregation as illustrated in Fig. 2e. Again, to rule out the effects of the eGFP tag, which possesses free cysteine as well, we ran the SDSPAGE with the tag-free bFGF, getting the same results (Supplementary Fig. 4c).

bFGF phase separates on cell surface mediated by heparan sulphate. As bFGF can undergo phase separation in presence of heparin in vitro, we further tested phase separation of bFGF on cell surface, where heparan sulphate is almost ubiquitous. We applied $500 \mathrm{nM}$ eGFP-bFGF to several cell lines, including mouse neuroblastoma cells (N2a), Chinese hamster ovary cells (CHOK1) and mouse embryonic fibroblast cells (MEF, BALB/C-3T3) cells to examine phase separation by confocal microscope. We found bright condensates formed on the edges of the cells incubated with eGFP-bFGF. Z-stack scanning confirmed existence of the condensates on the cell surface (Fig. 3a). As control, eGFP alone was applied to the cells and no condensate formation was observed (Supplementary Fig. 5). To further explore the location of the condensates, we stained the cell membrane and found that all the condensates existed out of the cell membrane (Supplementary Fig. 6a). We stained the early endosomes by the Rab4 antibody as well and no condensates found co-localised with the endosomes as they were washed away during processing, confirming that the condensates were extracellular. (Supplementary Fig. 6b). FRAP experiment showed fluorescence recovery, indicating liquid-like properties of the condensates (Fig. 3b). The results indicated that LLPS of bFGF is universal in various of cell lines. Moreover, larger number of droplets were observed on MEF cells compared with the other two strains, since HS was more abundant on MEF, indicating that HS induced LLPS. In cells 
N2a
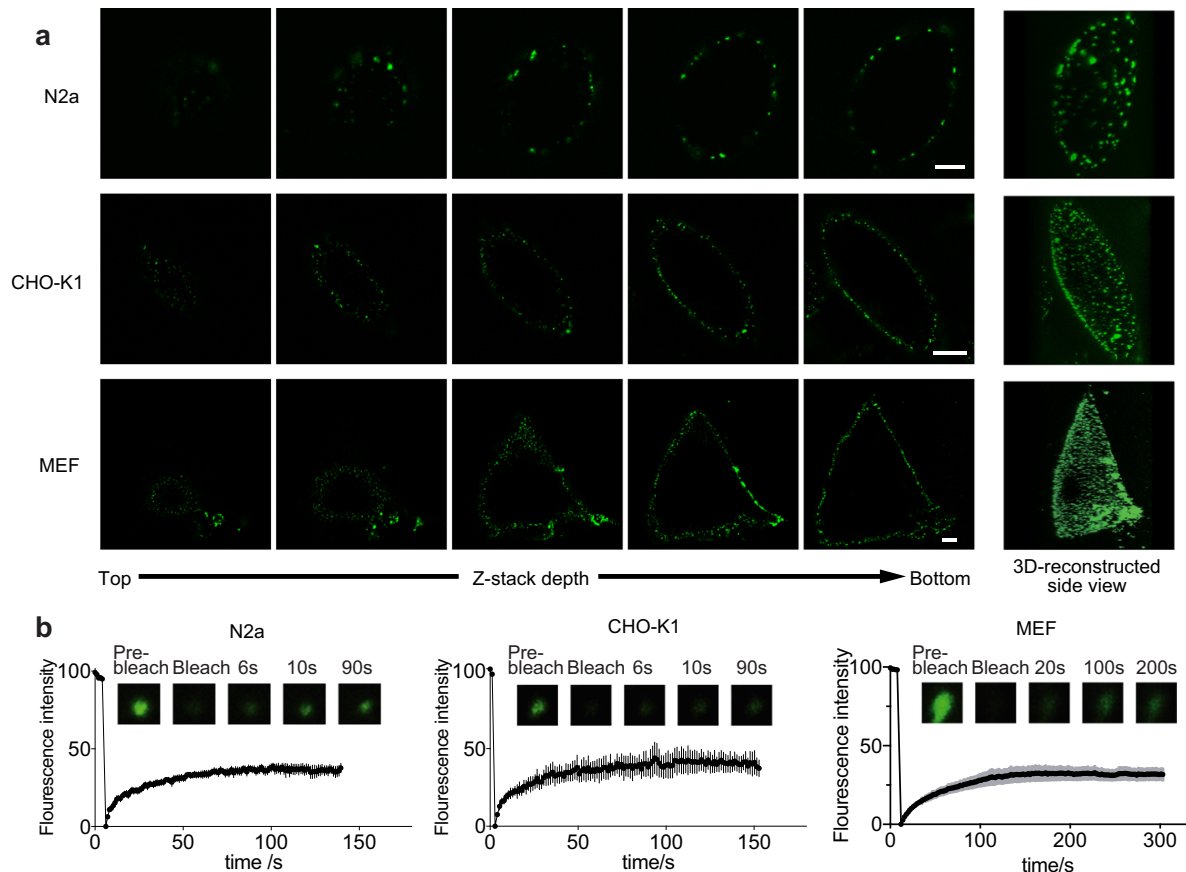

Z-stack depth
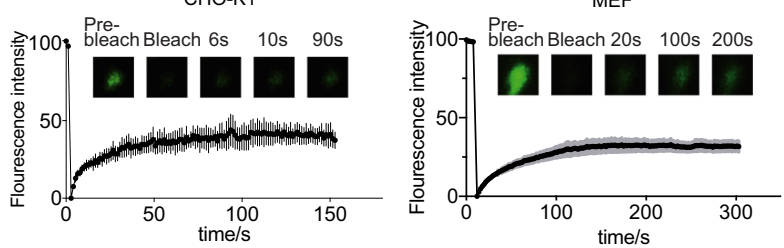

C

HS
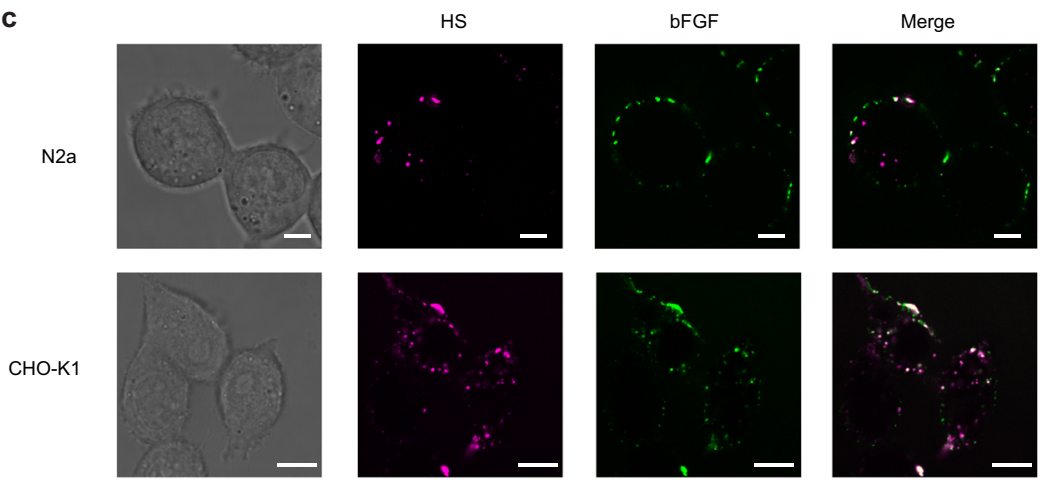

MEF
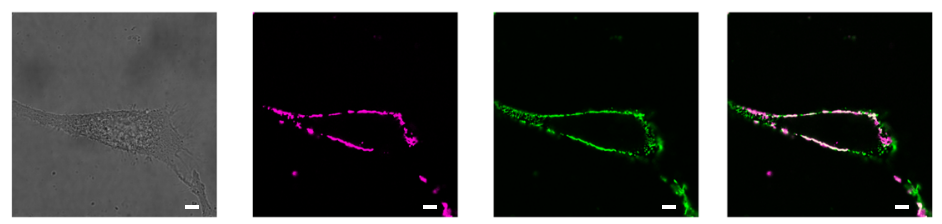

d

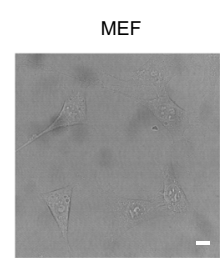

MEF treated with

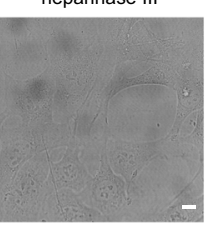

e

CHO-K1
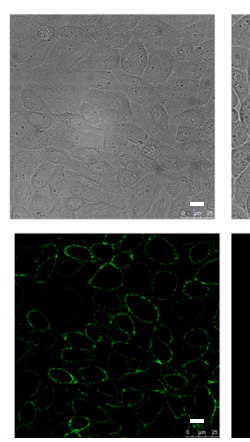

$\mathrm{CHO}-677$
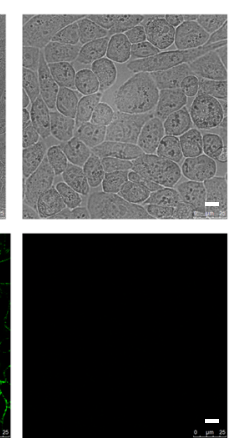

stained with anti-heparan sulphate antibody, eGFP-bFGF condensates overlapped significantly with heparan sulphate, suggesting that heparan sulfate likely mediated the formation of bFGF condensates (Fig. 3c). Further, we treated MEF cells with heparinase III to digest heparan sulphate on cell surface before adding eGFP-bFGF, and saw a significant decrease of the condensate amount (Fig. 3d). Moreover, in CHO-677, a cell line lacking heparan sulfate ${ }^{35}$, no condensate was detected, in contrast to the clear condensates observed on the CHO-K1 WT cells (Fig. 3e). These observations suggest that heparan sulphate is required for phase separation of bFGF on the cell surface. Phase separation increases effective concentration of bFGF within the 
Fig. 3 bFGF phase separates on cell surface with heparan sulphate. a Z-stack scanning of droplets formation on the cell surface. N2a, CHO-K1 or MEF cells incubated with eGFP-bFGF were imaged under confocal microscope with the Z-stack method, showing droplets condensation on the cell surface. Scale bar $=5 \mu \mathrm{m}$. b FRAP of the condensates formed by eGFP-bFGF. $n=4,3$ and 6 biologically independent samples for N2a, CHO-K1 and MEF cells respectively, data are presented as mean values \pm SEM. c Confocal microscopy images of heparan sulphate (HS, magenta) immunostaining and eGFP-bFGF (green) on N2a, CHO-K1 and MEF cells. Scale bar $=5 \mu \mathrm{m}$. $\mathbf{d}$ Confocal microscopy images of eGFP-bFGF phase separation on MEF cell surface with or without treatment with heparinase III. Scale bar $=10 \mu \mathrm{m}$. e Confocal microscopy images of eGFP-bFGF phase separation on the surface of wildtype CHO-K 1 and the HS deficient $\mathrm{CHO}-677$ cells. Scale bar $=10 \mu \mathrm{m}$.

phase and may promote its interaction with the receptor and affect downstream signalling ${ }^{3,6-8}$.

LLPS of bFGF facilitates signal transduction. FGFs exert activities through binding to their receptors, and heparan sulphate functions as a co-receptor to form HSPG-bFGF-FGFR ternary complex ${ }^{36,37}$. Since bFGF phase separate with HS on the cell surface, we suspected that the complex may co-condensate. FGFR antibody was used to label the receptor and the formation of FGFR and bFGF condensates was examined under confocal microscope (Fig. 4a). FGFR co-localised with bFGF condensates. Moreover, some bFGF condensates do not have FGFR signal, indicating the low affinity binding of bFGF with HSPG for its sequestering ${ }^{19,38}$. An experiment of doping the FGFR antibody to bFGF/HS mixture was conducted to rule out the antibody's effect on bFGF condensation or distribution (Supplementary Fig. 7). Next, we examined the impact of ternary phase separation on downstream signalling transduction. bFGF can activate multiple downstream signalling pathways, including Ras-ERK and PI 3Kinase-Akt pathway ${ }^{39}$. Here we examined the phosphorylated ERK as a marker for the signalling activation. We applied different concentrations of bFGF to MEF cells, monitored its phase separation under confocal microscope and quantified p-ERK level with Western blot. To determine the effect from LLPS, we added $500 \mu \mathrm{g} / \mathrm{mL}$ heparin to the cells with bFGF since the phase separation of bFGF can be inhibited by high concentration of heparin in vitro (see Fig. 1). This inhibition was observed on cell surface as well (Fig. 4b). As expected, the ERK phosphorylation increased along with bFGF through in a dose-dependent manner (Fig. 4c and d). When phase separation was inhibited by excess heparin or heparan sulphate was digested by heparinase, ERK phosphorylation was much diminished. We also explored the signalling at single-cell level by staining and quantifying phosphorylated ERK under the confocal microscopy (Supplementary Fig. 8), getting the same results. The results strongly suggested that phase separation is essential for activation of the downstream pathway of bFGF. Considering that bFGF exists in a low concentration $(1 \mathrm{ng} / \mathrm{mL})$ in human tissues ${ }^{40}$, there may be a mechanism to amplify its effects for the high efficacy. Though condensates at such a low concentration of bFGF was difficult to observe under microscope due to the limit of detection, the signalling data strongly suggested that the phase separation plays a role. We believe that LLPS driven by the interactions between bFGF and HSPG effectively increase the local concentration of bFGF around FGFR, augmenting growth signal transduction (Fig. 4e). Additionally, our findings also provided a model to explain the mysteries synergistic effects of heparin to bFGF, which heparin stimulates bFGF's effect at low concentrations while reduces it at high concentrations ${ }^{41,42}$.

Phase separation has gained recognition in multiple cellular processes, including membrane-less organelles formation and chromatin condensation. In these processes the intracellular environment is required to provide crowding effect and promote phase separation by multivalent weak interactions. Our study revealed phase separation activity on cell surface, where heparan sulphate serves as a platform to induce phase separation of bFGF.
In this process, bFGF is recruited and condensed into a distinct phase, which further facilitates the formation of bFGF-HSPGFGFR ternary complex to activate downstream signal transduction as well as stabilisation of bFGF. Phase separation on the cell surface thus represents a distinct mechanism for regulation of bFGF signalling.

Additionally, we briefly explored whether the heparin driven phase separation could occur with other heparin-binding growth factors. The acidic fibroblast growth factor (aFGF) was tested with different concentrations of heparin added. Both fluorescent images and turbidity demonstrated that LLPS occurs (Supplementary Fig. 9). Though with opposite charge compared with bFGF, aFGF possesses heparin-binding sites, which may provide weak interactions for phase separation. The results indicated that LLPS promoted by heparin may be universal for those heparinbinding growth factors.

Heparan sulphate is required for the interaction between a wide range of different cytokines and their receptors. HS mediated phase separation thus may apply to other signalling pathways and reshape the downstream response. Molecules like heparan sulphate can act as platforms to enrich signalling molecules and tune signal transduction. In addition, our findings also suggest that phase separation occurs not only in the intracellular environment but also in the extracellular environment, and has a potentially huge impact on extracellular physiology.

\section{Methods}

Protein expression and purification. Genes encoding His-eGFP or His-eGFP bFGF were constructed in pET-28a vector (Miao Ling Plasmid, China) and transformed into in E.coli (Transetta (DE3), TransGen Biotech, China) cells for expression. Cells were then grown to optical density of $0.9-1.2$ at $37^{\circ} \mathrm{C}$ and induced with $0.5 \mathrm{mM}$ isopropyl- $\beta$-d-thiogalactopyranoside (IPTG) at $16^{\circ} \mathrm{C}$ overnight. The cells were collected and lysed and protein was purified with His-Trap chelating column (GE Healthcare). The purified recombinant proteins were analysed by Coomassie-stained SDS-polyacrylamide gel electrophoresis (PAGE) and desalted into the final storage buffer $(100 \mathrm{mM}$ sodium phosphate, $\mathrm{pH} 7.2)$ by ÄKTA pure (General Electric, USA). Proteins were concentrated to $2 \sim 4 \mathrm{mg} / \mathrm{ml}$ using Ultra centrifugal filters. Aliquots were flash-frozen and stored at $-80^{\circ} \mathrm{C}$.

Cell culture. Mouse neuroblastoma (N2a) cells were cultured in complete medium containing 44.5\% Dulbecco's modified Eagle's medium (DMEM) $1 \times$ with glucose (4.5 g/L), 44.5\% Minimum Essential Medium (MEM) Alpha $1 \times, 10 \%$ fetal bovine serum (FBS), and $1 \%$ antibiotics (penicillin/streptomycin). Mouse embryonic fibroblast (MEF) cells were cultured in DMEM containing $10 \%$ FBS, $1 \%$ nonessential amino acid (NEAA) cell culture supplement and $1 \%$ antibiotics (penicillin /streptomycin). CHO-K1/CHO-677 cells were cultured in $\mathrm{F} 12 \mathrm{~K}$ medium containing $10 \%$ FBS and $1 \%$ antibiotics. All cells were cultured at $37^{\circ} \mathrm{C}$ with $5 \% \mathrm{CO}_{2}$ in a humidified incubator.

Imaging of bFGF phase separation in vitro. Purified eGFP-bFGF or tag-free bFGF (purchased from Sino Biological, China) were diluted to $0.5-1 \mathrm{mg} / \mathrm{ml}$ in assay buffer $\left(8 \mathrm{mM} \mathrm{Na}_{2} \mathrm{HPO}_{4}, 2 \mathrm{mM} \mathrm{KH}_{2} \mathrm{PO}_{4}, 136 \mathrm{mM} \mathrm{NaCl}\right.$ and $\left.2.6 \mathrm{mM} \mathrm{KCl}, \mathrm{pH} \mathrm{7.2}\right)$. For the phase separation of eGFP-bFGF, $5 \mu \mathrm{M}$ of the protein was mixed with increasing PEG- 8000 concentrations $(0-20 \% \mathrm{w} / \mathrm{v})$. For condensate formation with haperin, unless specified, $5 \mu \mathrm{M}$ of eGFP-bFGF or tag-free bFGF were mixed with heparin in the assay buffer. All operations were performed on ice. The mixed protein solution was immediately loaded into a 96-well plate and incubated for indicated time at the indicated temperature before imaging analysis. Images were captured with a Leica SP8 confocal microscopy with a $\times 100$ objective (oil immersion) and LAS X software 3.2. 
a

N2a

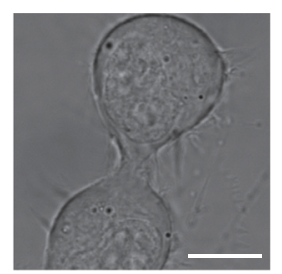

MEF

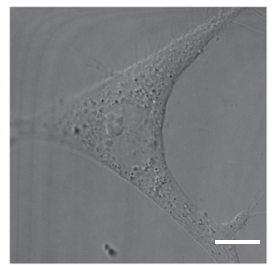

b
bFGF
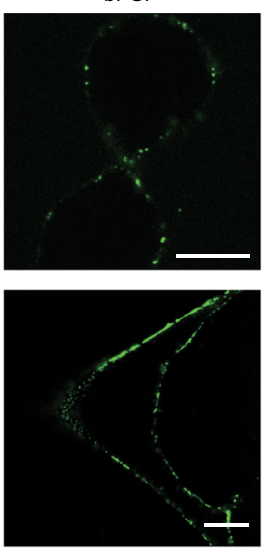

FGFR
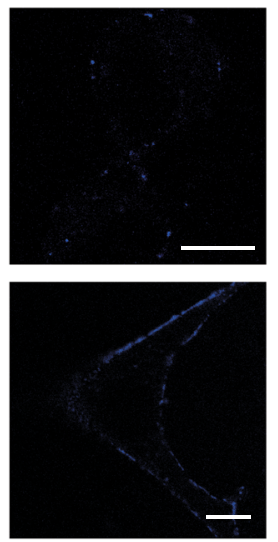

500
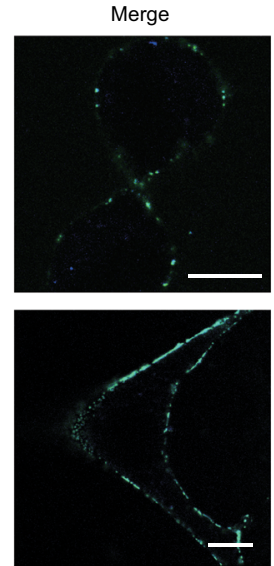

500

eated with

heparinase

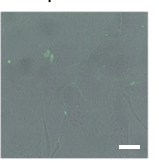

p-ERK

d

C

Heparinase
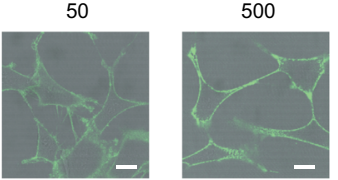

$500 \mu \mathrm{g} / \mathrm{mL}$ hepa

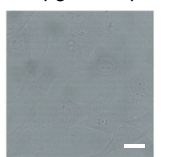

Heparin $500 \mu \mathrm{g} / \mathrm{mL}$

bFGF $n g / m L$

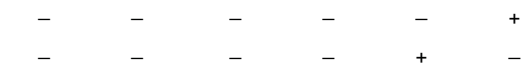

+
-

p-ERK1/2

0.1

10

10

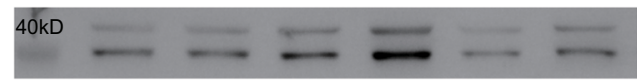

Vinculin

$130 \mathrm{kD}$
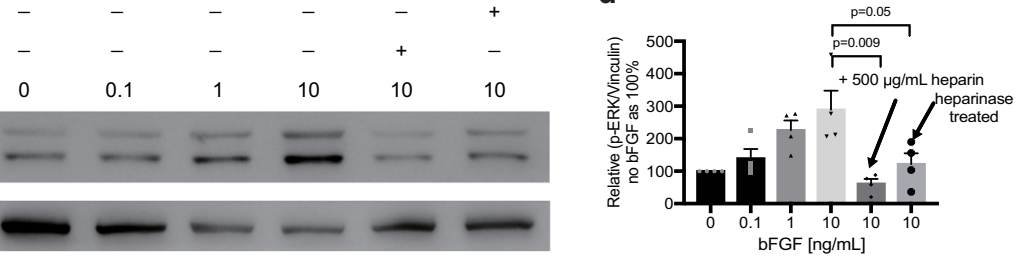

ERK
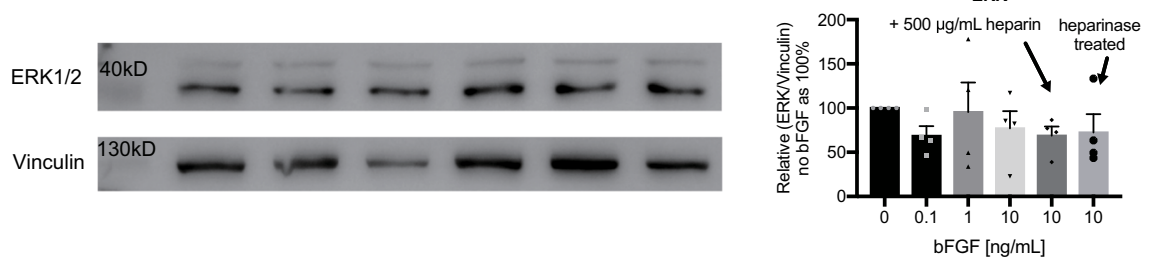

e

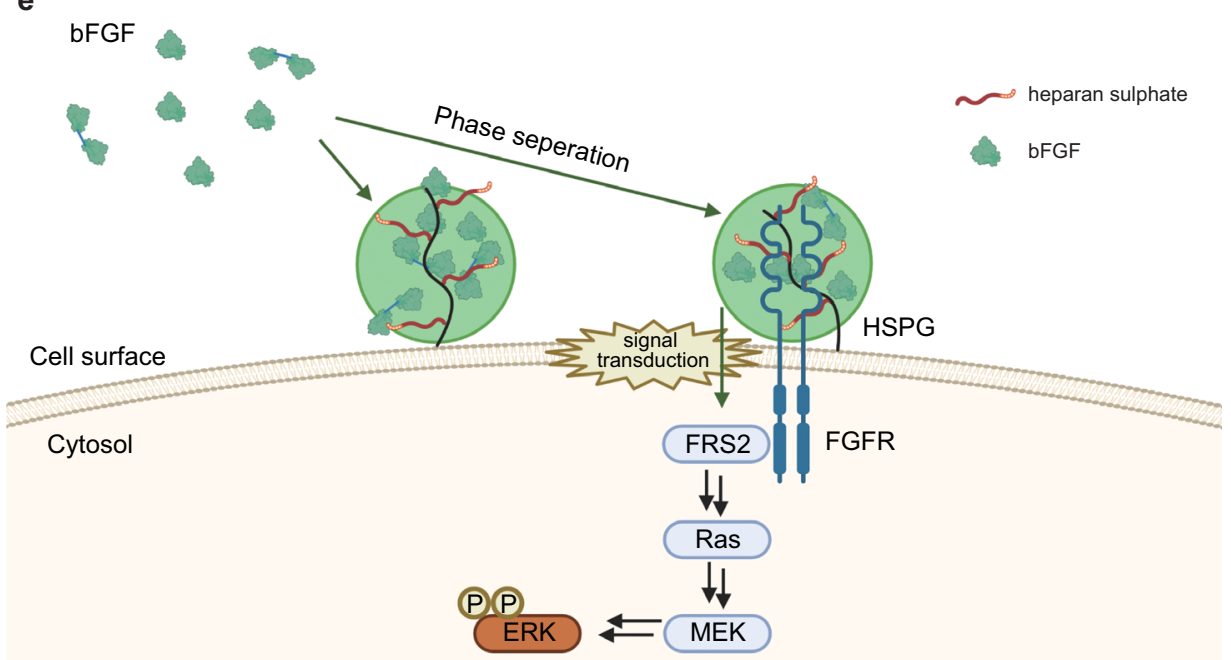

Turbidity assay. Proteins were prepared as described above. The protein solution was mixed with various concentrations of heparin $(0 \sim 5 \mu \mathrm{M})$ and PEG-8000 $(0-20 \% \mathrm{w} / \mathrm{v})$ in the assay. All operations were performed on ice. The mixed protein solution was immediately loaded into a 384 -well plate and incubated for 5-7 h at $4{ }^{\circ} \mathrm{C}$ before measuring. Turbidity was measured by absorption at $277 \mathrm{~nm}$ in $384-$ well plates using a SpectraMax M2 microplate reader (Molecular Devices). All samples were examined in triplicates $(N=3)$.

Analysis of bFGF oligomers by SDS-PAGE. eGFP-bFGF or tag-free bFGF $(5 \mu \mathrm{M})$ was mixed with various concentrations of heparin or de-O-sulphated heparin 
Fig. 4 The phase separation of bFGF triggers signalling transduction. a Confocal microscopy images of fluorescence-antibody FGFR (blue) and eGFPbFGF (green) on the surface of N2a or MEF cells. Scale bar $=10 \mu \mathrm{m}$. b Microscopy images showing phase separation of different concentrations of eGFPbFGF on MEF cells, with samples treated with $500 \mu \mathrm{g} / \mathrm{mL}$ heparin or heparinase. Scale bar $=20 \mu \mathrm{m}$. c Western blot showing ERK phosphorylation stimulated with bFGF at different concentrations, indicating the activation of Ras-ERK pathway. Added heparin $(500 \mu \mathrm{g} / \mathrm{mL}) \mathrm{inhibited}$ the downstream signalling. d Quantification of ERK expression and phosphorylation with bFGF phase separation. $n=4$ biologically independent samples, data are presented as mean values \pm SEM. Comparisons among groups were performed using two-tailed unpaired $t$ test. e Schematic illustration of the proposed model that bFGF phase separation along with HS on the cell surface for its capture and signalling activities.

(GlycoNovo Technologies, China) containing $1 \mathrm{mM} \mathrm{H}_{2} \mathrm{O}_{2}$ or $5 \mathrm{mM}$ DTT on ice. The samples were incubated at $4{ }^{\circ} \mathrm{C}$ in a final volume of $100 \mu \mathrm{L}$ of the assay buffer. After $10 \mathrm{~h}$, $\mathrm{N}$-ethyl maleimide ( $100 \mu \mathrm{M}$ final concentration) was used to block remaining thiols of cysteine residues. The samples $(30 \mu \mathrm{l})$ were mixed with $10 \mu \mathrm{l}$ of $4 \times$ SDS loading buffer with DTT (reducing) or without DTT (non-reducing) respectively, heated for $5 \mathrm{~min}$ at $100^{\circ} \mathrm{C}$, and analysed with SDS-PAGE.

Phase separation of bFGF on cell surface. Cells were plated onto an eight-well Lab-Tek chambered coverglass (Thermo Fisher Scientific) and cultured to $70 \%$. Before imaging, the medium was discarded, and the cells were washed with PBS twice. Then, the protein solution $(500 \mathrm{nM})$ was applied to the cells. Confocal microscopy was performed with an inverted Leica SP8 microscope, equipped with lasers for $405-\mathrm{nm}, 488-\mathrm{nm}, 552-\mathrm{nm}$ excitation. Images were acquired using a $100 \times$ objective and LAS X software 3.2.

Z-stack for Living Cell 3-D rendering. Three-dimensional reconstruction platform containing Z-stack were imaged with an inverted Leica SP8 microscope. Briefly, images were acquired using the $\times 100$ oil immersion lens, a pinhole of $1 \mathrm{AU}$, $488 \mathrm{~nm}$ laser with $10 \%$ laser power, followed by setting the starting position and end position of Z-stack, 100 200 Nr. of Steps or $1 \mu \mathrm{m}$ z-step size was selected. These $\mathrm{z}$-stack images were reconstructed with ImageJ 1.53.

\section{Fluorescence recovery after photobleaching of cell surface and in vitro con- densates. Samples of bFGF phase separation in solution or on cell surface were examined on an inverted microscope (LSM 780, Carl Zeiss, Germany) equipped with a confocal spinning disk unit (CSU-X1; Yokogawa, Tokyo, Japan) and a Zeiss $\times 100$ oil immersion lens. A field $(\sim 0.06 \mu \mathrm{m}$ for the formed droplet in vitro and $0.04 \mu \mathrm{m}$ for the punctate of cell surface) was bleached for $15 \mathrm{~ns}$ with $100 \%$ laser power of a $488-\mathrm{nm}$ or 405 lasers ( $1 \mathrm{AU}$ ) respectively. After being photobleached, images were acquired at a rate of $0.97 \mathrm{~s}$ (in solution) per frame or $1.26 \mathrm{~s}$ (on cell surface) for $500 \mathrm{~s}$. The fluorescent intensity of bleached area over time was cal- culated by Zeiss Zen 1.1. Signals were normalised with pre-bleached as $100 \%$ and $0 \mathrm{~s}$ after bleach as 0 . At least three FRAP curves were averaged to produce ach FRAP curve by Graphpad prism 7.0.}

Heparinase digestion. Cells were treated with heparinase III $(0.2 \mathrm{U} / \mathrm{mL}$, GlycoNovo Technologies, China) for $2-4 \mathrm{~h}$ at $37^{\circ} \mathrm{C}$. Then the cells were washed with phosphate-buffered saline (PBS) for three times before further treatments.

Immunofluorescence staining and live-cell imaging. The cells were plated on an eight-well Lab-Tek chambered coverglass at a density $2 \times 10^{4}-5 \times 10^{4}$ cells/well for N2a and CHO cells and 2000-4000 for MEF cells in $200 \mathrm{ul}$ medium and cultured for $24 \mathrm{~h}$, the culture medium was discarded, and cells were washed twice with PBS. For detection of cell surface heparan sulphate, the cells were incubated with an anti-HS antibody (10E4, USBiological, USA) diluted 1:100 in medium containing $1 \% \mathrm{BSA}$ for $2 \mathrm{~h}$ at $37^{\circ} \mathrm{C}$. After three washing steps with PBS, the cells were incubated with Alexa Fluor 647-conjugated secondary antibody (Jackson Immuno Research, USA, 1:200 dilution). For detection of cell surface FGFR1, the cells were stained with anti-FGFR1 antibody conjugated with Alexa Fluor 405 (Novus Biologicals, USA, 1:1000 dilution) for $1 \mathrm{~h}$ at $37^{\circ} \mathrm{C}$. The immune-stained cells were examined under a confocal laser scanning microscopy using an inverted Leica SP8 microscope, equipped with lasers for $405-\mathrm{nm}, 488-\mathrm{nm}, 552 \mathrm{~nm}, 638 \mathrm{~nm}$ excitation. Images were acquired using a $\times 100$ objective.

Western blot analysis. MEF cells (BALB/C-3T3) were plated on 6-well plates with a density $2 \times 10^{5}$ cells/well in $2 \mathrm{ml}$ medium and cultured for $48 \mathrm{~h}$. Then cells were switched into serum-free medium and cultured for $24 \mathrm{~h}$. Cells were next treated with $200 \mathrm{mIU} / \mathrm{mL}$ of Heparinase III (GlycoNovo Technologies, China) for $3 \mathrm{~h}$. After that, $500 \mu \mathrm{g} / \mathrm{mL}$ of Heparin and $0.1-10 \mathrm{ng} / \mathrm{mL}$ of eGFP-bFGF were added into the medium respectively, incubated for $1 \mathrm{~h}$. Cells were washed three times with PBS before collected and lysed with RIPA lysis buffer (Beyotime Biotechnology, China). The lysates were centrifuged for $10 \mathrm{~min}$ at $10,000 \mathrm{x} \mathrm{g}$ and the supernatants were used for Western blot. Anti-ERK1/2 antibody (Santa Cruz Biotechnology, USA, 1:200 dilution) and anti-p-ERK 1/2 (pT202/pY204.22 A, Santa Cruz Biotechnology, USA, 1:100 dilution) as used for the detection of total ERK and phosphorylated ERK. Horseradish peroxidase-linked anti mouse IgG (Beyotime
Biotechnology, China) was used as secondary antibody (1:2000 dilution). The signals were developed using BeyoECL Plus regent (Beyotime Biotechnology, China) and imaged with Chemiscope mini imaging system (CLINX, China). For protein loading control, vinculin antibody (Santa Cruz Biotechnology, USA, 1:200 dilution) was used. The results were analysed with ImageJ 1.53.

Bioactivity validation of the eGFP-bFGF. MEF (BALB/C-3T3) cell proliferation was measured using Cell Counting Kit-8 (Beyotime Biotechnology, China). Cells were seeded in a 96 -well plate $(3500 \mathrm{cell} / \mathrm{well} / 100 \mathrm{uL})$ in DMEM supplemented with $10 \% \mathrm{FBS}$ and allowed to adhere for $24 \mathrm{~h}$ at $37^{\circ} \mathrm{C}$. The medium was changed with serum-free DMEM containing various concentrations $(0,0.01,0.1,0.5,1,5,10$ and $100 \mathrm{ng} / \mathrm{mL}$ ) of either fusion recombinant eGFP-bFGF or commercial bFGF (SonoBiological, China). After $48 \mathrm{~h}$, the supernatants were removed, and $100 \mu \mathrm{L}$ of CCK- 8 working solution was added to each well for another $1 \mathrm{~h}$ at $37^{\circ} \mathrm{C}$. The CCK8 working solution was prepared with CCK-8 stock solution and DMEM medium at a 1:10 ratio. The optical density (OD) at $450 \mathrm{~nm}$ was measured using SpectraMax M2 microplate reader (Molecular Devices). Each group was performed with three replicates.

Analysis of the effect of salt concentration on bFGF phase separation. Purified proteins were diluted to $2 \mathrm{mg} / \mathrm{ml}$ and desalted into increasing $\mathrm{NaCl}$ concentration assay buffer $(0-500 \mathrm{mM})$. For eGFP-bFGF-heparin condensate formation, unless specified, $5 \mu \mathrm{M}$ of eGFP-bFGF were mixed with heparin in different $\mathrm{NaCl}$ concentration assay buffer. All operations were performed on ice. The mixed protein solution was immediately loaded into a 96-well plate and incubated for indicated time at the indicated temperature before imaging analysis. Images were captured with a Leica SP8 confocal microscopy with a $\times 100$ objective (oil immersion) and LAS X software 3.2.

Imaging of bFGF phase separation with polyU. eGFP-bFGF were diluted in assay buffer $\left(8 \mathrm{mM} \mathrm{Na}_{2} \mathrm{HPO}_{4}, 2 \mathrm{mM} \mathrm{KH}_{2} \mathrm{PO}_{4}, 136 \mathrm{mM} \mathrm{NaCl}\right.$ and $\left.2.6 \mathrm{mM} \mathrm{KCl}, \mathrm{pH} 7.2\right)$. $5 \mu \mathrm{M}$ of eGFP-bFGF were mixed with polyU in the assay buffer. All operations were performed on ice. The mixed protein solution was immediately loaded into a 96-well plate and incubated for indicated time at the indicated temperature before imaging analysis. Images were captured with a Leica SP8 confocal microscopy with a $\times 100$ objective (oil immersion).

Analysis of the effect of FGFR antibody on bFGF phase separation. In all, $5 \mu \mathrm{M}$ of eGFP-bFGF were mixed with heparin and increasing FGFR1 Antibody (Novus Biologicals, USA) concentrations $(0 \mu \mathrm{g} / \mathrm{mL}$ to $10 \mu \mathrm{g} / \mathrm{mL})$ in the assay buffer. All operations were performed on ice. The mixed protein solution was immediately loaded into a 96-well plate and incubated for indicated time at the indicated temperature before imaging analysis. Images were captured with a Leica SP8 confocal microscopy with a $\times 100$ objective (oil immersion) and LAS X software 3.2

Imaging of p-ERK in cells. MEF cells (BALB/C-3T3) cultured on coverslips (Solarbio, China) with a density $5 \times 10^{5}$ cells/well in $500 \mu \mathrm{L}$ medium and cultured for $48 \mathrm{~h}$. Then cells were switched into serum-free medium and cultured for $24 \mathrm{~h}$. Cells were next treated with $200 \mathrm{mIU} / \mathrm{mL}$ of Heparinase III (GlycoNovo Technologies, China) for $3 \mathrm{~h}$. After that, $500 \mu \mathrm{g} / \mathrm{mL}$ of Heparin and $0.1-10 \mathrm{ng} / \mathrm{mL}$ of eGFP-bFGF were added into the medium respectively, incubated for $1 \mathrm{~h}$. After washed three times with PBS, cells fixed with $4 \%$ paraformaldehyde for $20 \mathrm{~min}$ and permeabilized with $0.1 \%$ Triton X-100 for $5 \mathrm{~min}$ at room temperature (RT). The cells were placed in blocking buffer (3\% BSA in PBS) for $1 \mathrm{~h}$ following by washing and labelled with anti-p-ERK 1/2 (pT202/pY204.22 A, Santa Cruz Biotechnology, USA, 1:100 dilution) for overnight at $4{ }^{\circ} \mathrm{C}$. After three washing steps with PBS, the cells were incubated with Cy3-labelled Goat Anti-Mouse IgG(H $+\mathrm{L}$ ) (Beyotime Biotechnology, China, 1:500 dilution) antibody for $2 \mathrm{~h}$ at RT. The immune stained cells were examined under a confocal laser scanning microscopy using an inverted Leica SP8 microscope, equipped with lasers for 405-nm, 552-nm excitation. Images were acquired using a $\times 100$ objective and LAS $\mathrm{X}$ software 3.2 .

The fluorescence intensity of each cell was integrated using ImageJ and the corrected total cell fluorescence $(\mathrm{CTCF})$ was calculated as: $\mathrm{CTCF}=$ Integrated Density-(Area of selected cell X Mean fluorescence of background readings). 


\section{Early endosome and Phase separation of bFGF on cell surface. Cells were} plated onto an eight-well Lab-Tek chambered coverglass (Thermo Fisher Scientific) and cultured to around $70 \%$. Before imaging, the medium was discarded, and the cells were washed with PBS twice. Then, eGFP-bFGF protein solution (50 nM and $500 \mathrm{nM}$ ) was applied to the cells for $5 \mathrm{~min}$ at RT. After washed three times with PBS, cells fixed with $4 \%$ paraformaldehyde for $20 \mathrm{~min}$ and permeabilized with $0.1 \%$ Triton X-100 for $5 \mathrm{~min}$ at room temperature (RT). The resulting cells were placed in blocking buffer (3\% BSA in PBS) for $1 \mathrm{~h}$ followed by washing, and labelled with Anti-Rab4 Antibody- Early Endosome Marker (abcam, Britain, 1:170 dilution) for overnight at $4{ }^{\circ} \mathrm{C}$. After three washing steps with PBS, the cells were incubated with Cy3-labelled Goat Anti-Rabbit IgG(H + L) (Beyotime Biotechnology, China, 1:500 dilution) antibody for $2 \mathrm{~h}$ at RT. The immune stained cells were examined under a confocal laser scanning microscopy using an inverted Leica SP8 microscope, equipped with lasers for 405-nm, 488-nm, 552-nm excitation. Images were acquired using a $100 \times$ objective and LAS X software 3.2 .

Imaging of bFGF phase separation on cell membrane. Cells were plated onto an eight-well Lab-Tek chambered coverglass (Thermo Fisher Scientific) and cultured to around $70 \%$. Then, $5 \mu \mathrm{M}$ DilC18(3) (Beyotime Biotechnology, China) was applied to the cells for $15 \mathrm{~min}$ at RT. Before imaging, the cells were washed with PBS twice and the protein solution $(500 \mathrm{nM})$ was applied to the cells. Confocal microscopy was performed with an inverted Leica SP8 microscope, equipped with lasers for 405-nm, 488-nm, and 552-nm excitation. Images were acquired using a $\times 20$ or $\times 100$ objective and LAS $\mathrm{X}$ software 3.2.

Statistics and reproducibility. The fluorescent images, including Figs. 1a, e, h, 2a, d, 3a, c-e, and 4a, b; supplementary figs. 1a, 2, 3a, b, 4a, c, 5, 6a, b, 7, and 8a, in the manuscript represent at least three repeated experiments independently with similar results. The western blot in Fig. $4 \mathrm{c}$ represents for four repeated experiments independently with similar results.

Reporting summary. Further information on research design is available in the Nature Research Reporting Summary linked to this article.

\section{Data availability}

Authors can confirm that all relevant data are included in the paper and its supplementary information files. Source data are provided with this paper.

Received: 26 May 2021; Accepted: 9 February 2022;

Published online: 02 March 2022

\section{References}

1. Boeynaems, S. et al. Protein phase separation: a new phase in cell biology. Trends Cell Biol. 28, 420-435 (2018).

2. Zhang, H. et al. Liquid-liquid phase separation in biology: mechanisms, physiological functions and human diseases. Sci. China Life Sci. 63, 953-985 (2020).

3. Zeng, M. et al. Reconstituted postsynaptic density as a molecular platform for understanding synapse formation and plasticity. Cell 174, 1172-1187.e1116 (2018).

4. Li, P. et al. Phase transitions in the assembly of multivalent signalling proteins. Nature 483, 336-340 (2012).

5. Gibson, B. A. et al. Organization of chromatin by intrinsic and regulated phase separation. Cell 179, 470-484.e421 (2019).

6. $\mathrm{Su}, \mathrm{X}$. et al. Phase separation of signaling molecules promotes $\mathrm{T}$ cell receptor signal transduction. Science 352, 595-599 (2016).

7. Case, L. B., Zhang, X., Ditlev, J. A. \& Rosen, M. K. Stoichiometry controls activity of phase-separated clusters of actin signaling proteins. Science 363, 1093-1097 (2019).

8. Huang, W. Y. C. et al. A molecular assembly phase transition and kinetic proofreading modulate Ras activation by SOS. Science 363, 1098-1103 (2019).

9. Snead, W. T. \& Gladfelter, A. S. The control centers of biomolecular phase separation: how membrane surfaces, ptms, and active processes regulate condensation. Mol. Cell 76, 295-305 (2019).

10. Zhao, Y. G. \& Zhang, H. Phase separation in membrane biology: the interplay between membrane-bound organelles and membraneless condensates. Dev. Cell 55, 30-44 (2020).

11. Banjade S. \& Rosen M. K. Phase transitions of multivalent proteins can promote clustering of membrane receptors. Elife 3, e 04123 (2014).

12. Zeng, M. L. et al. Phase separation-mediated TARP/MAGUK complex condensation and AMPA receptor synaptic transmission. Neuron 104, 529 (2019).

13. Case, L. B., Ditlev, J. A. \& Rosen, M. K. Regulation of transmembrane signaling by phase separation. Annu. Rev. Biophys. 48, 465-494 (2019).
14. Banani, S. F., Lee, H. O., Hyman, A. A. \& Rosen, M. K. Biomolecular condensates: organizers of cellular biochemistry. Nat. Rev. Mol. Cell Biol. 18, 285-298 (2017)

15. Xue, S. et al. Low-complexity domain of U1-70K modulates phase separation and aggregation through distinctive basic-acidic motifs. Sci. Adv. 5, eaax5349 (2019).

16. Powers, C. J., McLeskey, S. W. \& Wellstein, A. Fibroblast growth factors, their receptors and signaling. Endocr. Relat. Cancer 7, 165-197 (2000).

17. Beenken, A. \& Mohammadi, M. The FGF family: biology, pathophysiology and therapy. Nat. Rev. Drug Discov. 8, 235-253 (2009).

18. Richardson, T. P., Trinkaus-Randall, V. \& Nugent, M. A. Regulation of basic fibroblast growth factor binding and activity by cell density and heparan sulfate. J. Biol. Chem. 274, 13534-13540 (1999).

19. Moscatelli, D. Basic fibroblast growth factor (bFGF) dissociates rapidly from heparan sulfates but slowly from receptors. Implications for mechanisms of bFGF release from pericellular matrix. J. Biol. Chem. 267, 25803-25809 (1992).

20. Eriksson, A. E., Cousens, L. S., Weaver, L. H. \& Matthews, B. W. Threedimensional structure of human basic fibroblast growth factor. Proc. Natl Acad. Sci. USA 88, 3441-3445 (1991).

21. Venkataraman, G. et al. Preferential self-association of basic fibroblast growth factor is stabilized by heparin during receptor dimerization and activation. Proc. Natl Acad. Sci. USA 93, 845-850 (1996).

22. Pak, C. W. et al. Sequence determinants of intracellular phase separation by complex coacervation of a disordered protein. Mol. Cell 63, 72-85 (2016).

23. Wang, Z., Zhang, G. \& Zhang, H. Protocol for analyzing protein liquid-liquid phase separation. Biophys. Rep. 5, 1-9 (2019).

24. Zeiger, A. S., Loe, F. C., Li, R., Raghunath, M. \& Van Vliet, K. J. Macromolecular crowding directs extracellular matrix organization and mesenchymal stem cell behavior. PLoS ONE 7, e37904 (2012).

25. Kaur, T. et al. Molecular crowding tunes material states of ribonucleoprotein condensates. Biomolecules 9, 71 (2019).

26. Burgess, W. H. \& Maciag, T. The heparin-binding (fibroblast) growth factor family of proteins. Annu. Rev. Biochem. 58, 575-606 (1989).

27. Ghosh, A., Mazarakos, K. \& Zhou, H. X. Three archetypical classes of macromolecular regulators of protein liquid-liquid phase separation. Proc. Natl Acad. Sci. USA 116, 19474-19483 (2019).

28. Maharana, S. et al. RNA buffers the phase separation behavior of prion-like RNA binding proteins. Science 360, 918-921 (2018).

29. Gospodarowicz, D. \& Cheng, J. Heparin protects basic and acidic FGF from inactivation. J. Cell Physiol. 128, 475-484 (1986).

30. Saksela, O., Moscatelli, D., Sommer, A. \& Rifkin, D. B. Endothelial cell-derived heparan sulfate binds basic fibroblast growth factor and protects it from proteolytic degradation. J. Cell Biol. 107, 743-751 (1988).

31. Pye, D. A., Vives, R. R., Turnbull, J. E., Hyde, P. \& Gallagher, J. T. Heparan sulfate oligosaccharides require 6-O-sulfation for promotion of basic fibroblast growth factor mitogenic activity. J. Biol. Chem. 273, 22936-22942 (1998).

32. Garg, H. G. et al. Sulfation patterns in heparin and heparan sulfate: effects on the proliferation of bovine pulmonary artery smooth muscle cells. Biochim. Biophys. Acta 1639, 225-231 (2003)

33. Sauer, D. G. et al. A two-step process for capture and purification of human basic fibroblast growth factor from E. coli homogenate: Yield versus endotoxin clearance. Protein Expr. Purif. 153, 70-82 (2019).

34. Zbinden, A. et al. Multivalent conjugates of basic fibroblast growth factor enhance in vitro proliferation and migration of endothelial cells. Biomater. Sci. 6, 1076-1083 (2018).

35. Esko, J. D., Rostand, K. S. \& Weinke, J. L. Tumor formation dependent on proteoglycan biosynthesis. Science 241, 1092-1096 (1988).

36. Escobar Galvis, M. L. et al. Transgenic or tumor-induced expression of heparanase upregulates sulfation of heparan sulfate. Nat. Chem. Biol. 3, 773-778 (2007).

37. Zhang, Z., Coomans, C. \& David, G. Membrane heparan sulfate proteoglycansupported FGF2-FGFR1 signaling: evidence in support of the "cooperative end structures" model. J. Biol. Chem. 276, 41921-41929 (2001).

38. Folkman, J. et al. A heparin-binding angiogenic protein-basic fibroblast growth factor-is stored within basement membrane. Am. J. Pathol. 130, 393-400 (1988).

39. Teven, C. M., Farina, E. M., Rivas, J. \& Reid, R. R. Fibroblast growth factor (FGF) signaling in development and skeletal diseases. Genes Dis. 1, 199-213 (2014).

40. Tripathi, R. C., Borisuth, N. S. \& Tripathi, B. J. Detection, quantification, and significance of basic fibroblast growth factor in the aqueous humor of man, cat, dog and pig. Exp. Eye Res. 54, 447-454 (1992).

41. Lundin, L. et al. Selectively desulfated heparin inhibits fibroblast growth factor-induced mitogenicity and angiogenesis. J. Biol. Chem. 275, 24653-24660 (2000).

42. Newman, D. R., Li, C. M., Simmons, R., Khosla, J. \& Sannes, P. L. Heparin affects signaling pathways stimulated by fibroblast growth factor-1 and -2 in type II cells. Am. J. Physiol. Lung Cell Mol. Physiol. 287, L191-L200 (2004). 


\section{Acknowledgements}

This work was supported by funding from National Key R\&D Program of China (2021YFC2103900 to S-Z. L), National Natural Science Foundation of China (22077010 and 91853116 to S-Z. L), Natural Science Foundation of Beijing, China (2204088 to S.X.), Young Scientists Fund of the National Natural Science Foundation of China (21907007 to S.X.), the Fundamental Research Funds for the Central Universities (buctrc201920 to S.X., BAIC201814 to J.L.) and the Swedish Research Council (to J.L.). We thank Dr. Yunxiao Zhang, Dr. Shuibing Chen and Dr. Yongxiang Chen for helping to edit the manuscript. The illustrations of mechanism were created with BioRender.com, and we thank Dr. Mingjia Yu for helping to preparing the illustrations.

\section{Author contributions}

S.X. and S.-Z.L. designed the experiments, analysed the data, and wrote the manuscript. F.Z. and T.Z. performed the experiments and analysed the data. H.Z. helped the cell culture and assays. X.W. and L.C. helped preparing the protein. S.-Z.L., S.X. and J.L. supervised the whole project.

\section{Competing interests}

The authors declare no competing interests.

\section{Additional information}

Supplementary information The online version contains supplementary material available at https://doi.org/10.1038/s41467-022-28765-z.

\section{Correspondence and requests for materials should be addressed to Shi-Zhong Luo.}

Peer review information Nature Communications thanks Martin Götte and the other, anonymous, reviewer(s) for their contribution to the peer review of this work.

Reprints and permission information is available at http://www.nature.com/reprints

Publisher's note Springer Nature remains neutral with regard to jurisdictional claims in published maps and institutional affiliations.

\section{(c) (1)}

Open Access This article is licensed under a Creative Commons Attribution 4.0 International License, which permits use, sharing, adaptation, distribution and reproduction in any medium or format, as long as you give appropriate credit to the original author(s) and the source, provide a link to the Creative Commons license, and indicate if changes were made. The images or other third party material in this article are included in the article's Creative Commons license, unless indicated otherwise in a credit line to the material. If material is not included in the article's Creative Commons license and your intended use is not permitted by statutory regulation or exceeds the permitted use, you will need to obtain permission directly from the copyright holder. To view a copy of this license, visit http://creativecommons.org/ licenses/by/4.0/.

(c) The Author(s) 2022 